\title{
A PHYTOSOCIOLOGICAL ANALYSIS ON KIZILKUYU WILDLIFE DEVELOPMENT AREA (ŞANLIURFA/TURKEY)
}

\author{
Ömer Faruk, KAYA \\ Harran University, Şanlıurfa, Turkey, phytosociologist@gmail.com
}

\begin{abstract}
Bordered by Taurus Mountains in the north and Syria - Iraq arid climate zone in the south, Southeastern Anatolia has wide steppe lands. As a conclusion to the phytosociological research done in accordance with Braun-Blanquet (1965) method in Kizılkuyu Wildlife Development Area, which was founded in these steppe lands, three associations belonging to steppe vegetation were found. Two of these associations are new to scientific world. Identified associations were generally found in slightly stony areas inappropriate to agricultural practices. The study area is in Irano-Turanian phytogeographic region and found in C7 square in Grid System (Davis 1965 - 1985).
\end{abstract}

\section{INTRODUCTION}

Southeastern Anatolian steppes cover the northern part of "fertile crescent" and they are significantly barren when compared with Central Anatolian steppes. Forest lower bound begins at $700-850$ meters in the borders of the steppe. A wide area of Southeastern Anatolian steppe, excluding the Diyarbakır Basin, is a plateau area formed of prairies and flat hills following each other (Atalay, 1994).

This region, which is also known as Upper Mesopotamia, is a geographical transition zone between the Arabian Platform and the folded rocky mountains in the north where plateaus are widest. The plateau elevation is approximately 1000 meters in the north, and it decreases to about 500-600 meters in the south (Anonymous, 1986).

Şanliurfa province, in where the study area is found, shows the characteristics of Pliocene period, which is the last period of Cenozoic era. The land was formed together with the old world. Not affected much by side pressures and eruptions occurred between the end of Cenozoic era and the beginning of Quaternary era, Şanliurfa elevated on the hard rock body and formed folds in places. Northeastern parts of Şanlıurfa province, especially Siverek, Hilvan and Viranşehir are formed of basalts erupted from Karacadağ. A part of the province where the study area is also found is covered by limestone formations (Anonymous, 2011).
Because of climate, topography, and bedrock differences, there are different major soil groups in Şanlıurfa. Lithosol soils which are in the azonal soil group found in high inclination areas, brown soils, and colluvial soils, and the wasteland is formed of rocks and rubbles are seen in the study area.

Kizilkuyu Wildlife Development Area, found on Mesopotamian plains, covers an area of 20504 hectares, which is in southwest of Şanliurfa. Its geographical position is between $36^{\circ} 90^{\prime} 00^{\prime \prime}$ and $37^{\circ} 8^{\prime} 00^{\prime \prime}$ in northern latitudes and $38^{\circ}$ $30^{\prime} 40^{\prime \prime}$ and $38^{\circ} 50^{\prime} 00^{\prime \prime}$ in eastern longitudes (Yeniyurt et al., 2009) (Fig. 1).

The study area is bordered by İkizce village in the east, Yanıkçögür village in the west, Keberli village in the north and Altınbaşak village in the south (Fig. 2).

Average altitude of the study area is 600 meters and rocks and stones cover a big part of it. Highest hill is Dikilitaş hill, with an altitude of 764 meters. The altitude decreases gradually from north to south (Yeniyurt et al., 2009).

There are no streams flowing throughout the year in the study area. Intermittent streams extending through south between the hills form rift alluvial plains on the south part of the area. The hills composed of a bedrock that is formed of limestone are covered by a thin layer of soil and sparse vegetation. Northern part of the study area is nonarable, generally seems as a natural steppe. Southern parts, however, are comparably arable with wide lands of cultivation (Yeniyurt et al., 2009). 


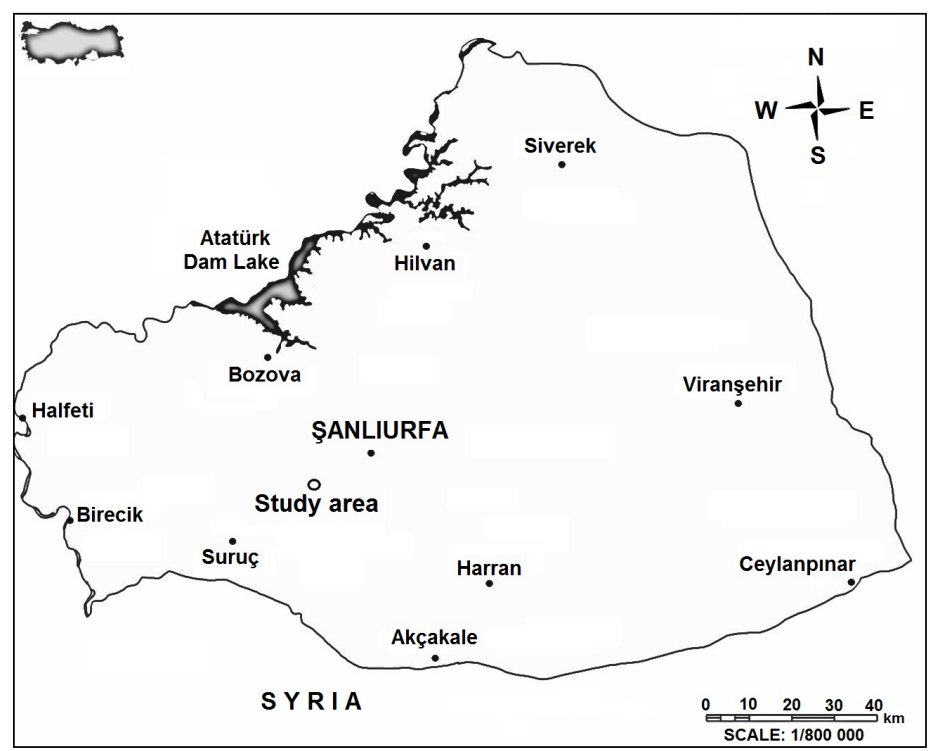

Figure 1. Geographical map of Şanlıurfa.

\section{METHODS}

Studies by Davis (1965-1985), Davis et al. (1988), Güner et al. (2000), Kaya and Ertekin (2009), Kaya (2010a; 2011a) and Kaya et al. (2011b)

Climate of the study area was evaluated in accordance with the meteorological data provided by the weather station of Şanliurfa (Anonymous, 2010). The analysis of soil samples taken from places where can represent the associations in the study area was done in accordance with Tüzüner (1990) methods.

In this research, Braun - Blanquet's "Floristic Unit System" (1965) and cover-abundance scale elaborated in accordance with this system by Frey and Lösch (1998) were used for vegetation analysis. According to this scale; ( $\mathrm{r}=1$ individual, also rare outside the relevé, small plant); $+=2-5$ (small) individuals, cover $<5 \% ; 1=6-50$ individuals, cover $<5 \%$; or few larger individuals (often given as 1-5) with a cover up to
$5 \% ; 1 \mathrm{~m}=$ many individuals $(>50)$, cover $<5 \% ; 2 \mathrm{a}=$ cover 5 $12.4 \% ; 2 \mathrm{~b}=$ cover $12.5-25 \% ; 3=$ cover $25-50 \% ; 4=$ cover 50 $75 \% ; 5=$ cover $75-100 \%)$.

In the determination of relevés width, "minimal area" method was followed and this value was determined as $50 \mathrm{~m}^{2}$ for steppe vegetation. For the naming of the new associations, International code of phytosociological nomenclature" was referred (Weber et al., 2000). For the classification of steppe vegetation associations, studies of Quézel et al. (1973), Akman et al. (1985) with Kaya and Ketenoğlu (2010) were referred.

Comparison of identified associations with similar associations in the respect of floristic composition was made by using Sørensen (1948) similarity index $\left[\mathrm{C}_{\mathrm{s}}=(2 \times \mathrm{C}\right.$ x 100) / $(\mathrm{A}+\mathrm{B})]$

$\left(\mathrm{C}_{\mathrm{s}}=\right.$ similarity coefficient, $\mathrm{A}$ is the number of species found in site $\mathrm{A} ; \mathrm{B}$ is the number of species in site $\mathrm{B}$ and $\mathrm{C}$ is the number of species shared by the two sites).

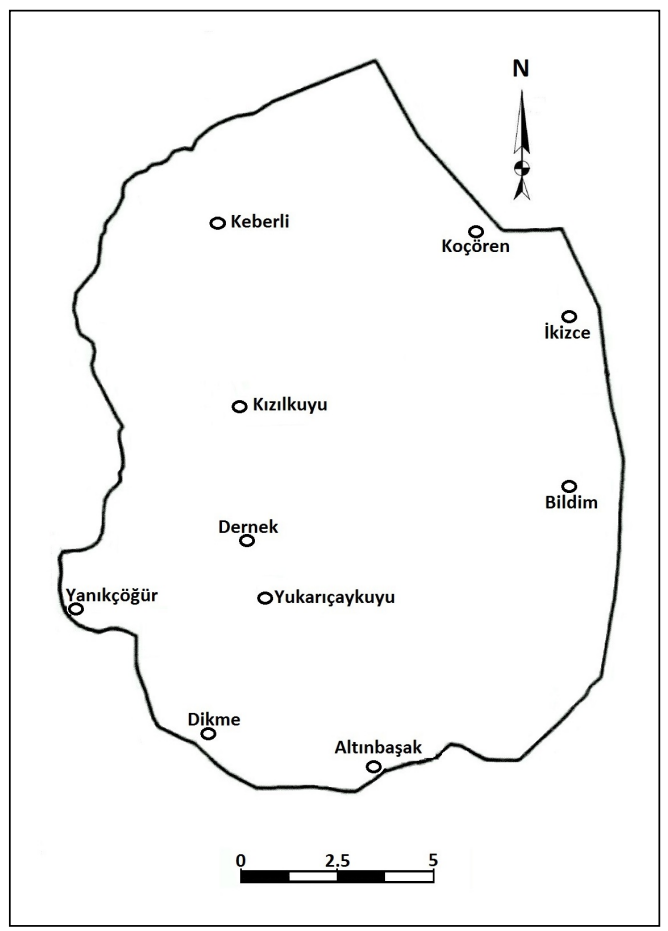

Figure 2. Local map of Kizilkuyu wildlife development area. 


\section{RESULTS AND DISCUSSIONS}

Three associations belonging to steppe vegetation were identified in the study area.

\section{Eryngio cretico - Asphodelinetum brevicaulis ass.nova}

Holotypus: relevé 5, $540 \mathrm{~m}$, cover $80 \%, 50 \mathrm{~m}^{2}$, Table 1

The association is characterized by Asphodeline brevicaulis subsp. brevicaulis var. brevicaulis and Eryngium creticum. The association is occurred by a single layer of vegetation, which is formed by xerophytes of $25-60 \mathrm{~cm}$ height, with a cover rate of $75-80 \%$. Floristic composition of the association is not substantial. Many species in the association have low cover rates. Main reason for this is the fact that the area is being used as a grazing land by the neighbouring villages.

Asphodeline brevicaulis subsp. brevicaulis var. brevicaulis, dominating the physiognomy of the association, is an Eastern Mediterranean element, which spreads to Western and Eastern Anatolia's rocky and stony areas. This association around the dumping ground, that is about $2 \mathrm{~km}$ north of İkizce village, which deploys in northeast of the study area, shows prevalence on limestone bedrock at about $500-550 \mathrm{~m}$ altitude and $5^{\circ}-10^{\circ}$ slope.
The texture class of the association soils is clay and loamy and shows slight alkaline reaction $(\mathrm{pH} 7.87$ - 7.93). Lime level is $17.31 \%-35.66 \%$ and organic matter level differs between $1.28 \%-1.29 \%$.

\section{Centaurea virgatae - Convolvuletum oxysepali ass.nova}

Holotypus: relevé $17,636 \mathrm{~m}$, cover $75 \%, 50 \mathrm{~m}^{2}$, Table 2

Characteristic taxa of the association are Convolvulus dorycnium subsp. oxysephalus and Centaurea virgata. Dominant species of the association is Convolvulus dorycnium subsp. oxysephalus is an Eastern Mediterranean element generally showing prevalence around Southern and Eastern Anatolia. This association is deployed in the stony area among Kizılkuyu, Güzelkuyu and Keberli villages, in average height of $610-650 \mathrm{~m}, 2^{\circ}-5^{\circ}$ slope.

The association is formed of a single layer and has a cover rate of $55-75 \%$ and height of $5-40 \mathrm{~cm}$. Texture class of soils is clay-loamy. The soil has $\mathrm{pH}$ values around $7.90-7.92$ and is slight alkaline. Lime level is around $22.99 \%-25.34 \%$ so the limiest soil of the study area belongs to this association's soil. Organic matter level differs between $1.18 \%-1.40 \%$

Table 1. Eryngio cretico - Asphodelinetum brevicaulis ass.nova

\begin{tabular}{|c|c|c|c|c|c|c|c|c|c|c|c|c|}
\hline & Relevé no & 21 & 22 & 23 & 24 & 25 & 26 & 27 & 28 & 29 & 30 & \\
\hline & Size of plot $\left(\mathrm{m}^{2}\right)$ & 50 & 50 & 50 & 50 & 50 & 50 & 50 & 50 & 50 & 50 & \\
\hline & Parent rock & LS & LS & LS & LS & LS & LS & LS & LS & LS & LS & E \\
\hline & Inclination $\left({ }^{\circ}\right)$ & 5 & 5 & 5 & 10 & 10 & 7 & 10 & 8 & 5 & 5 & 產 \\
\hline & Altitude (m) & 536 & 540 & 540 & 5505 & 540 & 535 & 542 & 547 & 538 & 543 & ڤั \\
\hline & Exposition & W & W & $\mathrm{E}$ & $\mathrm{E}$ & SE & $\mathrm{E}$ & $\mathrm{E}$ & W : & NW & $\mathrm{E}$ & \\
\hline & Coverage (\%) & 75 & 75 & 75 & 75 & 80 & 75 & 75 & 75 & 75 & 75 & \\
\hline \multicolumn{13}{|l|}{ LF } \\
\hline & Differential and characteristic species of the association & & & & & & & & & & & \\
\hline $\mathrm{H}$ & Asphodeline brevicaulis subsp. brevicaulis var. brevicaulis & 4 & 4 & 4 & 4 & 5 & 4 & 4 & 3 & 4 & 4 & $\mathrm{~V}$ \\
\hline \multirow[t]{2}{*}{$\mathrm{H}$} & Eryngium creticum & 1 & & 1 & & 1 & + & + & 1 & + & & IV \\
\hline & Characteristic species of Astragalo erythrotaeni-Gundelic & n ar & mate & & & & & & & & & \\
\hline $\mathrm{H}$ & Gundelia tournefortii var. armata & + & & & + & + & & + & & + & & III \\
\hline \multirow[t]{2}{*}{$\mathrm{H}$} & Torilis leptocarpa & & + & + & & & + & & & & & II \\
\hline & Characteristic species of Onobrychido armenae-Thymeta & lia le & cost & tomi & & & & & & & & \\
\hline $\mathrm{H}$ & Centaurea virgata & & + & & & + & & & + & & + & II \\
\hline \multirow[t]{2}{*}{$\mathrm{H}$} & Paronychia kurdica subsp. kurdica var. kurdica & & & & & + & & & & + & & I \\
\hline & Characteristic species of Astragalo microcephali-Bromete & a to & men & telli & & & & & & & & \\
\hline $\mathrm{Ch}$ & Teucrium polium & 1 & & & + & 1 & & 1 & & + & + & III \\
\hline Th & Xeranthemum annиum & + & + & & & + & & + & & & & II \\
\hline $\mathrm{H}$ & Ajuga chamaepitys subsp. chia var. chia & + & & & + & & + & & & + & & II \\
\hline \multirow[t]{2}{*}{ Th } & Picnomon acarna & & & + & & & & + & & & & I \\
\hline & Companions & & & & & & & & & & & \\
\hline Th & Trifolium speciosum & + & & + & & + & + & & + & & & III \\
\hline Th & Trifolium stellatum var. stellatum & & 1 & & + & 1 & + & 1 & + & & & III \\
\hline Th & Ziziphora taurica subsp. taurica & + & & + & & 1 & + & & & 1 & + & III \\
\hline Th & Tordylium hasselquistiae & & + & + & & + & & + & & + & & III \\
\hline Th & Valerianella vesicaria & & & + & & + & . & & & + & & II \\
\hline Th & Alyssum strigosum subsp. strigosum & + & & + & & + & & & + & & & II \\
\hline $\mathrm{H}$ & Astragalus diphtherites var. diphtherites & & & + & & + & & + & & & & II \\
\hline $\mathrm{H}$ & Echinops orientalis & & & 1 & + & + & & & & & & II \\
\hline $\mathrm{Th}$ & Aegilops triuncialis subsp. triuncialis & + & & & + & & & 1 & & + & & II \\
\hline Th & Echinaria capitata & & & & & + & & + & & & 1 & II \\
\hline $\mathrm{Th}$ & Ziziphora capitata & 1 & & & & & & & + & 1 & + & II \\
\hline $\mathrm{H}$ & Phlomis bruguieri & + & 1 & & + & & + & & & & & II \\
\hline $\mathrm{H}$ & Sanguisorba minor subsp. magnolii & & & + & + & & & & + & & & II \\
\hline Th & Taeniatherum caput-medusae subsp. crinitum & & + & & & + & & & + & & + & II \\
\hline $\mathrm{H}$ & Verbascum kotschyi & + & & & + & & & & & + & & II \\
\hline $\mathrm{H}$ & Trigonella coelesyriaca & & & + & & + & & + & & & + & II \\
\hline Th & Scandix stellata & + & & & + & & & & & + & & II \\
\hline $\mathrm{Ch}$ & Thymbra spicata var. spicata & & + & & & & + & & & + & & II \\
\hline Th & Bromus tectorum & & & & + & + & + & & & + & & II \\
\hline Th & Trigonella mesopotamica & & + & & & + & & + & & & & II \\
\hline $\mathrm{G}$ & Gladiolus atroviolaceus & & + & & & & + & & + & & & II \\
\hline $\mathrm{H}$ & Achillea aleppica subsp. aleppica & & + & + & & & + & & & & + & II \\
\hline Th & Trifolium boissieri & & & + & + & & + & & & & + & II \\
\hline Th & Hippocrepis unisiliqua subsp. unisiliqua & & + & & + & & & & + & & & II \\
\hline Th & Holesteum umbellatum var. umbellatum & & + & & & + & & & & + & & II \\
\hline $\mathrm{Th}$ & Trigonella monantha subsp. monantha & & & + & + & + & & & & & & II \\
\hline Th & Valerianella kotschyi & & + & & + & + & & & & + & & II \\
\hline $\mathrm{Th}$ & Avena sterilis subsp. sterilis & & + & & & & + & & & + & & II \\
\hline $\mathrm{H}$ & Hordeum bulbosum & & & 1 & & + & & + & & & + & II \\
\hline Th & Trifolium pilulare & + & & & & + & & & & & & I \\
\hline $\mathrm{G}$ & Hyacinthella nervosa & + & & & & & & & & & & I \\
\hline G & Ixiolirion tataricum subsp. montanum & & + & + & & & & & & & & I \\
\hline Th & Trifolium pauciflorum & + & & & & + & & & & & & I \\
\hline $\mathrm{H}$ & Aristolochia bottae & & + & & & + & & & & & & I \\
\hline Th & Geranium rotundifolium & & + & & & & & & & & + & I \\
\hline Th & Senecio vernalis & & & & + & & & & + & & & I \\
\hline G & Gagea reticulata & & & & & + & & & + & & & I \\
\hline $\mathrm{H}$ & Salvia palaestina & + & & & & & + & & & & & I \\
\hline
\end{tabular}


Table 2. Centaurea virgatae - Convolvuletum oxysepali ass.nova

\begin{tabular}{|c|c|c|c|c|c|c|c|c|c|c|c|c|}
\hline & Relevé no & 11 & 12 & 13 & 14 & 15 & 16 & 17 & 18 & 19 & 20 & \multirow{7}{*}{ 总 } \\
\hline & Size of plot $\left(\mathrm{m}^{2}\right)$ & 50 & 50 & 50 & 50 & 50 & 50 & 50 & 50 & 50 & 50 & \\
\hline & Parent rock & LS & LS & LS & LS & LS & LS & LS & LS & LS & LS & \\
\hline & Inclination $\left(^{\circ}\right)$ & 2 & 2 & 3 & 5 & 2 & 2 & 2 & 5 & 3 & 3 & \\
\hline & Altitude (m) & \multicolumn{10}{|c|}{620621617630625635636640638635} & \\
\hline & Exposition & W & W & $\mathrm{W}$ & $\mathrm{E}$ & $\mathrm{E}$ & $\mathrm{E}$ & $\mathbf{E}$ & $\mathrm{E}$ & $\mathrm{E}$ & $\mathrm{E}$ & \\
\hline & Coverage $(\%)$ & 55 & 65 & 55 & 65 & 70 & 70 & 75 & 70 & 55 & 65 & \\
\hline \multirow[t]{2}{*}{$\mathbf{L F}$} & & & & & & & & & & & & \\
\hline & \multicolumn{4}{|c|}{ Differential and characteris tic species of the association } & & & & & & & & \\
\hline $\mathrm{H}$ & Convolvulus dorycnium subsp. oxysephalus & 3 & 3 & 4 & 5 & 3 & 4 & 5 & 4 & 5 & 4 & $\mathrm{~V}$ \\
\hline \multirow[t]{2}{*}{$\mathrm{H}$} & Centaurea virgata & & & 1 & 1 & + & & $\mathbf{1}$ & + & & + & III \\
\hline & \multicolumn{7}{|c|}{ Characteris tic species of Astragalo erythrotaeni-Gundelion armatae } & & & & & \\
\hline \multirow[t]{2}{*}{$\mathrm{H}$} & Gundelia tournefortii var. armata & & & + & + & & & + & & & + & II \\
\hline & \multicolumn{7}{|c|}{ Characteristic species of Onobrychido armenae-Thymetalia lecostomi } & & & & & \\
\hline $\mathrm{H}$ & Noaea mucronata subsp. mucronata & + & + & & & & + & + & & & & III \\
\hline $\mathrm{H}$ & Achillea wilhelmsii & & & + & & & & + & & & + & II \\
\hline \multirow[t]{2}{*}{$\mathrm{H}$} & Paronychia kurdica subsp. kurdica var. kurdica & & + & & + & & & & & + & & II \\
\hline & Characteristic species of As tragalo microcephal & i-Br & ome & tea & tom & entel & & & & & & \\
\hline $\mathrm{Ch}$ & Teucrium polium & & 1 & & + & 1 & & $\mathbf{1}$ & & & + & III \\
\hline Th & Medicago rigidula var. rigidula & & 1 & & + & & + & + & + & & & III \\
\hline $\mathrm{H}$ & Ajuga chamaepitys subsp. chia var. chia & & & + & & & & + & & & + & II \\
\hline $\mathrm{H}$ & Poa bulbosa & + & & & + & & & & & & + & II \\
\hline $\mathrm{G}$ & Allium scorodoprasum subsp. scorodoprasum & & + & & & & & + & & + & & II \\
\hline Th & Xeranthemum annuum & + & & & & & & + & & & & I \\
\hline & Companions & & & & & & & & & & & \\
\hline Th & Echinaria capitata & + & + & & 1 & + & 1 & + & & 1 & & IV \\
\hline Th & Trifolium pilulare & + & + & 1 & & + & & $\mathbf{1}$ & + & & + & IV \\
\hline Th & Erophila verna subsp. verna & & & 1 & + & 1 & 1 & + & & & & III \\
\hline Th & Lagoecia cuminoides & 1 & & + & & + & & $\mathbf{1}$ & + & & 1 & III \\
\hline $\mathrm{H}$ & Trigonella coelesyriaca & + & & & & + & & + & + & & + & III \\
\hline $\mathrm{H}$ & Sanguisorba minor subsp. magnolii & + & & & + & + & & & + & + & & III \\
\hline $\mathrm{H}$ & Astragalus diphtherites var. diphtherites & & + & & & & + & + & & + & + & III \\
\hline Th & Avena sterilis subsp. sterilis & & + & & + & & & + & + & & + & III \\
\hline Th & Scandix pecten-veneris & & & + & + & & + & + & + & & & III \\
\hline Th & Aegilops triuncialis subsp. triuncialis & & 1 & & + & & & + & + & & & II \\
\hline $\mathrm{H}$ & Vinca major subsp. major & & & + & & & & & & & + & II \\
\hline $\mathrm{G}$ & Gynandriris sisyrinchium & & + & & & & + & & + & & + & II \\
\hline $\mathrm{H}$ & Scorzonera mollis subsp. szowitzii & + & + & & & & + & & + & & & II \\
\hline $\mathrm{H}$ & Onosma molle & + & & & + & & & + & & & & II \\
\hline $\mathrm{H}$ & Phlomis kurdica & & + & & & & 1 & & + & & + & II \\
\hline $\mathrm{H}$ & Erodium ciconium & + & & & & & + & & & + & & II \\
\hline Th & Legousia speculum-veneris & & 1 & & + & & & + & + & & & II \\
\hline Th & Trigonella monantha subsp. monantha & & & + & & 1 & & & + & & 1 & II \\
\hline Th & Geranium rotundifolium & & + & & & & & + & & + & & II \\
\hline $\mathrm{H}$ & Ranunculus asiaticus & & & + & & & & + & + & & & II \\
\hline Th & Hippocrepis unisiliquosa subsp. unisiliquosa & & & + & & + & 1 & & & & + & II \\
\hline Th & Trifolium boissieri & & & + & + & & + & & + & & & II \\
\hline $\mathrm{H}$ & Hordeum bulbosum & & + & & & 1 & + & & & & & II \\
\hline Th & Crepis sancta & & + & + & & & + & & & + & & II \\
\hline Th & Euphorbia petiolata & & & & + & & & & + & & + & II \\
\hline Th & Erodium gruinum & + & & & + & & & + & & & + & II \\
\hline $\mathrm{H}$ & Cerastium kotschyi & & + & + & & & + & & + & & & II \\
\hline $\mathrm{Ch}$ & Thymbra spicata var. spicata & & + & & + & & & + & & & + & II \\
\hline $\mathrm{H}$ & Phlomis bruguieri & 1 & & + & & & + & & & & + & II \\
\hline $\mathrm{H}$ & Micromeria myrtifolia & & & + & & & + & & & & + & II \\
\hline Th & Trigonella mesopotamica & & & + & & & & + & & & & I \\
\hline $\mathrm{H}$ & Salvia syriaca & + & & + & & & & & & & & I \\
\hline $\mathrm{H}$ & Pimpinella eriocarpa & + & & & + & & & & & & & I \\
\hline Th & Campanula strigosa & & + & & & & & & & & & I \\
\hline $\mathrm{H}$ & Onopordum bracteatum & & & & & + & & & & & & I \\
\hline $\mathrm{H}$ & Andrachne telephioides & & & + & & & & + & & & & I \\
\hline Th & Androsace maxima & & & & + & & & & & & & I \\
\hline Th & Alyssum minus var. minus & & + & & & & & + & & & & I \\
\hline $\mathrm{G}$ & Biarum carduchorum & & & & + & & & + & & & & I \\
\hline $\mathrm{H}$ & Convolvulus betonicifolius subsp. peduncularis & + & & & + & & & & & & & I \\
\hline Th & Crupina crupinastrum & & + & & & 1 & & + & & & & I \\
\hline $\mathrm{H}$ & Umbilicus horizontalis var. intermedius & & + & & & + & & & & & & I \\
\hline $\mathrm{H}$ & Tragopogon longirostis var. longirostis & & & + & & & & + & & & & I \\
\hline
\end{tabular}

\section{Phlomidetum kurdico - bruguieri Kaya 2011 (Table 3)}

This association generally spreads on limestone bedrock, at around $550-590 \mathrm{~m}$ height and around $0^{\circ}-3^{\circ}$ slope. The lime level of the soil is around $11.50-27.11 \%$ and the organic matter level is around $1.10-1.11 \%$ and it has $\mathrm{pH}$ values around $7.91-7.93$ and slight alkaline. The texture class of soils is clay - loamy.

This association is widest and most scattered association in the study area. Its best deployed is observed at $500-550 \mathrm{~m}$ height, between Dernek and Yukarıçaykuyu villages. 
Table 3. Phlomidetum kurdico - bruguieri Kaya 2011

\begin{tabular}{|c|c|c|c|c|c|c|c|c|c|c|c|c|}
\hline & Relevé no & 1 & 2 & 3 & 4 & 5 & 6 & 7 & 8 & 9 & 10 & \multirow{7}{*}{ 总 } \\
\hline & Size of plot $\left(\mathrm{m}^{2}\right)$ & 50 & 50 & 50 & 50 & 50 & 50 & 50 & 50 & 50 & 50 & \\
\hline & Parent rock & LS & LS & LS & LS & LS & LS & LS & LS & LS & LS & \\
\hline & Inclination $\left({ }^{\circ}\right)$ & $\mathbf{0}$ & 2 & 1 & 0 & 3 & 0 & 2 & 0 & 3 & 0 & \\
\hline & Altitude (m) & \multicolumn{10}{|c|}{561563565570570565580580560570} & \\
\hline & Exposition & $\mathbf{N}$ & NW & $\mathrm{SE}$ & $\mathrm{S}$ & $\mathrm{N}$ & W & SE & $\mathrm{S}$ & $\mathrm{S}$ & W & \\
\hline & Coverage $(\%)$ & 75 & 70 & 75 & 70 & 70 & 65 & 65 & 70 & 65 & 70 & \\
\hline \multirow[t]{2}{*}{$\mathbf{L F}$} & & & & & & & & & & & & \\
\hline & \multicolumn{4}{|c|}{ Differential and characteristic species of the association } & & & & & & & & \\
\hline $\mathrm{H}$ & Phlomis bruguieri & 5 & 4 & 4 & 4 & 3 & 3 & 3 & 4 & 4 & 3 & V \\
\hline \multirow[t]{2}{*}{$\mathrm{H}$} & Phlomis kurdica & + & + & 1 & + & 1 & + & + & 1 & + & + & $\mathrm{V}$ \\
\hline & \multicolumn{7}{|c|}{ Characteristic species of Astragalo erythrotaeni-Gundelion armatae } & & & & & \\
\hline \multirow[t]{2}{*}{$\mathrm{H}$} & Gundelia tournefortii var. armata & + & & & & + & + & & & & & II \\
\hline & \multicolumn{7}{|c|}{ Characteristic species of Onobrychido armenae-Thymetalia lecostomi } & & & & & \\
\hline $\mathbf{H}$ & Marrubium parviflorum subsp. parviflorum & + & + & & & + & & + & & & & II \\
\hline $\mathrm{H}$ & Paronychia kurdica subsp. kurdica var. kurdica & + & & & + & & + & & + & & & II \\
\hline \multirow[t]{2}{*}{$\mathrm{H}$} & Centaurea virgata & & & + & + & & & + & & & & II \\
\hline & Characteristic species of Astragalo microcepha & i-Br & omet & tea t & tome & ente & & & & & & \\
\hline $\mathrm{Ch}$ & Teucrium polium & + & 1 & & & & + & & & 1 & + & III \\
\hline Th & Medicago radiata & + & & & 1 & + & + & & 1 & & 1 & III \\
\hline Th & Lamium amplexicaule & + & + & & & + & & + & & & 1 & III \\
\hline $\mathrm{H}$ & Poa bulbosa & & & + & + & & 1 & + & + & & & III \\
\hline Th & Bromus japonicus subsp. japonicus & & & + & + & & & & + & & & II \\
\hline Th & Trifolium campestre & + & & & & + & & & & + & & II \\
\hline Th & Xeranthemum annuum & + & + & & & + & & & & & + & II \\
\hline $\mathbf{H}$ & Ajuga chamaepitys subsp. chia var. chia & & & + & & & & & + & & & I \\
\hline & Companions & & & & & & & & & & & \\
\hline Th & Aegilops triuncialis subsp. triuncialis & + & & + & 1 & + & & 1 & + & & 1 & IV \\
\hline Th & Valerianella vesicaria & $\mathbf{1}$ & + & & & 1 & & + & & + & & III \\
\hline Th & Trifolium tomentosum & $\mathbf{1}$ & & + & + & 1 & & & & & + & III \\
\hline $\mathbf{H}$ & Onobrychis kotschyana & & + & & + & & & + & & + & + & III \\
\hline Th & Avena sterilis subsp. sterilis & $\mathbf{1}$ & + & & & 1 & & & + & + & & III \\
\hline Th & Echinaria capitata & + & & & + & & + & + & & & + & III \\
\hline Th & Filago pyramidata & + & + & & + & & & & 1 & & + & III \\
\hline Th & Coronilla scorpioides & & + & & & & + & & + & & + & II \\
\hline Th & Papaver dubium subsp. dubium & + & & & & & & + & & & & II \\
\hline Th & Trifolium speciosum & & & & + & & & & & + & & II \\
\hline $\mathrm{H}$ & Torilis leptocarpa & + & & & & & & + & & & + & II \\
\hline Th & Cicer echinospermum & & + & & & & + & + & & & & II \\
\hline $\mathbf{H}$ & Achillea aleppica subsp. aleppica & + & & + & & & & & & + & & II \\
\hline Th & Trifolium boissieri & + & & + & & & + & + & & & & II \\
\hline Th & Onobrychis aequidentata & & & + & & & & + & & & + & II \\
\hline $\mathbf{H}$ & Tragopogon longirostris var. longirostris & + & & & 1 & & & & + & & & II \\
\hline $\mathbf{H}$ & Dianthus strictus var. strictus & & + & & 1 & & & & + & & & II \\
\hline $\mathbf{H}$ & Convolvulus betonicifolius subsp. peduncularis & + & & & & + & & & & + & + & II \\
\hline Th & Tordylium hasselquistiae & + & & & & & + & & 1 & & & II \\
\hline Th & Ziziphora capitata & $\mathbf{1}$ & + & & & & 1 & & & 1 & & II \\
\hline $\mathrm{G}$ & Ornithogalum narbonense & & + & & & & + & & + & & & II \\
\hline Th & Rhagadiolus angulosus & & & + & & & & + & & & + & II \\
\hline $\mathbf{H}$ & Astragalus suberosus subsp. suberosus & + & & & & + & & & & + & & II \\
\hline $\mathbf{H}$ & Salvia multicaulis & & + & & & & + & & + & & + & II \\
\hline $\mathbf{H}$ & Hordeum bulbosum & + & & & + & & 1 & & & & & II \\
\hline $\mathbf{H}$ & Onosma sericeum & + & & & + & & + & & & + & & II \\
\hline Th & Alyssum strigosum subsp. strigosum & + & & + & & & & + & + & & & II \\
\hline $\mathbf{H}$ & Astragalus diphtherites var. diphtherites & & + & & & & + & & & + & + & II \\
\hline $\mathbf{H}$ & Sanguisorba minor subsp. magnolii & & & & + & + & & & & + & & II \\
\hline $\mathbf{H}$ & Aristolochia bottae & & + & & + & & & & & + & & II \\
\hline Th & Lens orientalis & + & & & & & + & + & & & & II \\
\hline Th & Cephalaria setosa & & & & & & & & + & + & & I \\
\hline $\mathbf{H}$ & Andrachne telephioides & & & + & & & & & & & & I \\
\hline Th & Crupina crupinastrum & + & & & + & & & & & & & I \\
\hline $\mathbf{H}$ & Echinops orientalis & & + & & & & & & + & & & I \\
\hline Th & Trifolium cherleri & & & & & + & 1 & & & & & I \\
\hline Th & Trifolium pilulare & & & & + & & & & + & & & I \\
\hline $\mathrm{G}$ & Gagea reticulata & + & & & & & & & + & & & I \\
\hline Th & Ceratocephalus falcatus & $\mathbf{1}$ & & & & & + & & & & & I \\
\hline Th & Trigonella monspeliaca & & & & + & & & + & & & & I \\
\hline $\mathbf{H}$ & Salvia palaestina & & + & & & & & + & & & & I \\
\hline
\end{tabular}

Another area covered by this association is in the south of the study area, on the hills deploys south of Çakırlar village, where is out of study area's boundaries. The association shows weak presence in the hills between Koçören and Güzelkuyu villages, at 620 - $640 \mathrm{~m}$ height and between Koşma and Dernek villages, at 550 - $560 \mathrm{~m}$ height.

It is possible to encounter with the characteristic taxa of this association, Phlomis bruguieri and Phlomis kurdica in almost every part of the study area as small groups formed of $5-10$ members.

Phlomis bruguieri is an Irano-Turanian element specifying the physiognomy of the association. This taxon spreads generally in South-eastern Anatolia.

This association was previously identified in a phytosociological research conducted by Kaya (2011a) in Germuş Mountain. When this association in the study area is compared with Phlomidetum kurdico - bruguieri identified in 
Germuş Mountain, in accordance with Sørensen's (1948) similarity index, the similarity of floristic composition is calculated as $53.5 \%$.

According to the data obtained from chemical analysis of soil samples from the study area, all the associations show prevalence on the clay - loamy soil. Such soil type has the optimum physical features for the vegetation. Soil types in the study area are slight alkaline in terms of $\mathrm{pH}$ value and in the class of saltless soils in terms of electrical conductivity (EC) (Table 4).

Table 4. Chemical analysis of the soils taken from the study area. (CL: clay-loamy)

\begin{tabular}{|c|c|c|c|c|c|c|c|c|c|}
\hline Associations & $\begin{array}{c}\text { Relevé } \\
\text { No }\end{array}$ & $\begin{array}{c}\text { Saturation } \\
(\%)\end{array}$ & $\begin{array}{c}\mathrm{EC} \\
\mathrm{ds} / \mathrm{m}\end{array}$ & $\mathbf{p H}$ & $\begin{array}{c}\mathrm{CaCO}_{3} \\
(\%)\end{array}$ & $\begin{array}{c}\mathbf{P}_{2} \mathbf{O}_{5} \\
\text { (kg/da) }\end{array}$ & $\begin{array}{c}\mathrm{K}_{2} \mathrm{O} \\
(\mathrm{kg} / \mathrm{da})\end{array}$ & $\begin{array}{c}\text { Organic } \\
\text { matter } \\
(\%)\end{array}$ & Texture \\
\hline \multirow{2}{*}{$\begin{array}{c}\text { Eryngio cretico- } \\
\text { Asphodelinetum } \\
\text { brevicaulis }\end{array}$} & 5 & 64 & 0,962 & 7,87 & 17,31 & 1,20 & 65,72 & 2,20 & \multirow{6}{*}{$\mathrm{CL}$} \\
\hline & 9 & 60 & 0,864 & 7,93 & 35,66 & 1,04 & 47,71 & 2,23 & \\
\hline \multirow{2}{*}{$\begin{array}{c}\text { Centaurea virgatae } \\
\text { - Convolvuletum } \\
\text { oxysepali }\end{array}$} & 12 & 54 & 0,909 & 7,92 & 22,99 & 2,01 & 130,55 & 2,42 & \\
\hline & 17 & 56 & 0,948 & 7,90 & 25,34 & 2,09 & 116,00 & 2,03 & \\
\hline \multirow{2}{*}{$\begin{array}{c}\text { Phlomidetum } \\
\text { kurdico - bruguieri }\end{array}$} & 21 & 60 & 0,944 & 7,93 & 11,50 & 1,52 & 92,44 & 1,91 & \\
\hline & 28 & 61 & 0,947 & 7,91 & 27,11 & 1,85 & 86,15 & 1,90 & \\
\hline
\end{tabular}

A large extent of Turkish soil is composed of lime soil (Gedikoğlu, 1990). Analysed association soils show great difference in terms of lime levels. While Phlomidetum kurdico - bruguieri spread on medium or high lime level soil, Centaurea virgatae - Convolvuletum oxysepali and Eryngio cretico - Asphodelinetum brevicaulis spread on high and very high lime level soils.

Turkish soil lacks phosphorus in a major part (Eyüpoğlu, 1999). Analysis conducted on association soils support this data. The phosphorus levels are low in all three syntaxa.

A major part of Turkish soil has potassium in abundance (Eyüpoğlu, 1999). Conclusion made from the analysis of association soils show that, even though potassium levels change in the soil of three syntaxa, the general level is high. Potassium support for plant efficiency is not required.

Turkish soil generally lacks organic matter (Eyüpoğlu, 1999). According to the results of the analysis, while organic matter levels are low in Phlomidetum kurdico - bruguieri, Centaurea virgatae - Convolvuletum oxysepali and medium in Eryngio cretico - Asphodelinetum brevicaulis .

As a result; in consequence of the conducted soil analysis, it is realized that the soil parameters are in optimal percentage for the growth of plants. According to this conclusion, weak vegetation is caused by other factors apart from soil such as overgrazing, land clearing, temperature, bare rocks etc.

South-eastern Anatolian climate shows characteristics of a continental steppe climate. Lowest temperatures and precipitation are features of winter, while summer is arid and hot. These facts put forward the existence of a vague Mediterranean type precipitation regime. As there are no altitude differences in the parts of the study area, amount of precipitation does not change. In the study area, amount of precipitation is high in winter and low in summer. Precipitation regime is W. Sp. A. Sm. (winter, spring, autumn, summer) thus, this signalizes Eastern Mediterranean precipitation regime, type 1 (Table 5).

Table 5. Climatic data of the station in the study area.

\begin{tabular}{|c|c|c|c|c|c|c|c|c|c|c|c|c|c|c|}
\hline $\begin{array}{c}\text { Meteorological } \\
\text { elements }\end{array}$ & $\begin{array}{c}\text { Periods } \\
\text { (years) }\end{array}$ & I & II & III & IV & V & VI & VII & VIII & IX & X & XI & XII & Annual \\
\hline $\begin{array}{c}\text { Mean } \\
\text { temperature } \\
\left({ }^{0} \mathrm{C}\right)\end{array}$ & 41 & 5.6 & 6.9 & 11.0 & 16.1 & 22.3 & 28.2 & 31.9 & 31.1 & 26.8 & 20.2 & 12.5 & 7.3 & 18.3 \\
\hline $\begin{array}{c}\text { Max. mean } \\
\text { temperature }\end{array}$ & 41 & 10.1 & 11.9 & 16.7 & 22.3 & 28.8 & 34.7 & 38.7 & 38.2 & 33.9 & 27.0 & 18.4 & 11.9 & 24.3 \\
\hline $\begin{array}{c}\text { Min. mean } \\
\text { temperature }\end{array}$ & 41 & 2.2 & 3.0 & 6.3 & 10.7 & 15.8 & 21.0 & 24.5 & 24.0 & 20.2 & 15.0 & 8.3 & 4.0 & 12.9 \\
\hline $\begin{array}{c}\text { Mean } \\
\text { precipitation } \\
\text { (mm) }\end{array}$ & 41 & 73.0 & 69.1 & 65.0 & 47.0 & 25.4 & 3.6 & 0.7 & 1.1 & 3.0 & 25.6 & 45.9 & 72.9 & 432.3 \\
\hline $\begin{array}{c}\text { Mean relative } \\
\text { humidity (\%) }\end{array}$ & 41 & 69.6 & 66.7 & 61.4 & 57.5 & 46.1 & 35.0 & 32.3 & 35.5 & 38.1 & 47.7 & 61.0 & 70.2 & 51.7 \\
\hline
\end{tabular}

\section{CONCLUSIONS}

Gazelles (Gazella subgutturosa), were regarded as endangered species in 1970's and were under protection. The species were reproduced semi-wildly at the reproduction station in Ceylanpinar town and for the release of surplus members to wildlife; the study area was announced as K1z1lkuyu Wildlife Development Area in 2006. This area is in one of the 305 key biodiversity areas of Turkey, the “Akçakale Key Biodiversity Area” (Eken et al., 2006). 
Gazelles left in the study area (86 individuals) grew rapidly and in present, there are 500 individuals. In addition to sheep, goats and cows belonging to the villages in the study area, the gazelles increased the grazing pressure on the existing vegetation. Progressively, this pressure increases and the study area is on the brink of exceeding the carrying capacity of a population. The proof to this is the existence of bare lands without vegetation in some parts of the study area.

South-eastern Anatolian steppe vegetation has been exposed to anthropological factors for years. This causes the vegetation to reproduce through regressive succession. The steppe vegetation of K1z1lkuyu Wildlife Development Area can be regarded as malacophyllous steppe physiognomically. The area is floristically poor because of its topographical structure, which causes lack of isolated areas and overgrazing pressure.

Most common herbaceous plants in the study area are Paoceae family members such as Poa bulbosa, Avena sterilis subsp. sterilis, Aegilops triuncialis subsp. triuncialis, Bromus japonicus subsp. japonicus; tragacanthic plants characterized, thorny taxa such as Astragalus spp., Onosma spp., Onopardum sp., Echinops sp. etc.; and broad-leaved, perennial plants such as Phlomis spp., Verbascum spp.
There are no forest formations in the study area, but rarely, phanerophytes such as Rosa canina, Cerasus microcarpa subsp. tortuosa and Ficus carica subsp. rupestris are seen. Apart from the existing steppe species, vegetation belonging to ruderal and segetal taxa are also seen. The reason for this is the presence of cultivated areas and transportation roads.

As a result of the factors such as the existence of wide, stony areas and the usage of the land as a grazing point, the floristic composition of identified associations is composed of taxa not preferred by animals. In other words, we can say that there is a zootic climax in the study area by the effect of these biotic factors.

When the chronology of taxa in identified associations are analysed, it is seen that Irano - Turanian elements are higher in percentage. This result supports the idea that, Southeastern Anatolia, which was also included in Zohary's (1973) study area, exists in the Irano - Turanian phytogeographic region. Additionally, the existence of Eastern Mediterranean elements (Convolvulus dorycnium subsp. oxysephalus and Centaurea virgata) dominating the physiognomy of the two associations, shows that the study area is a microclimatic zone, where features of Mediterranean climate are observed (Figure 3).

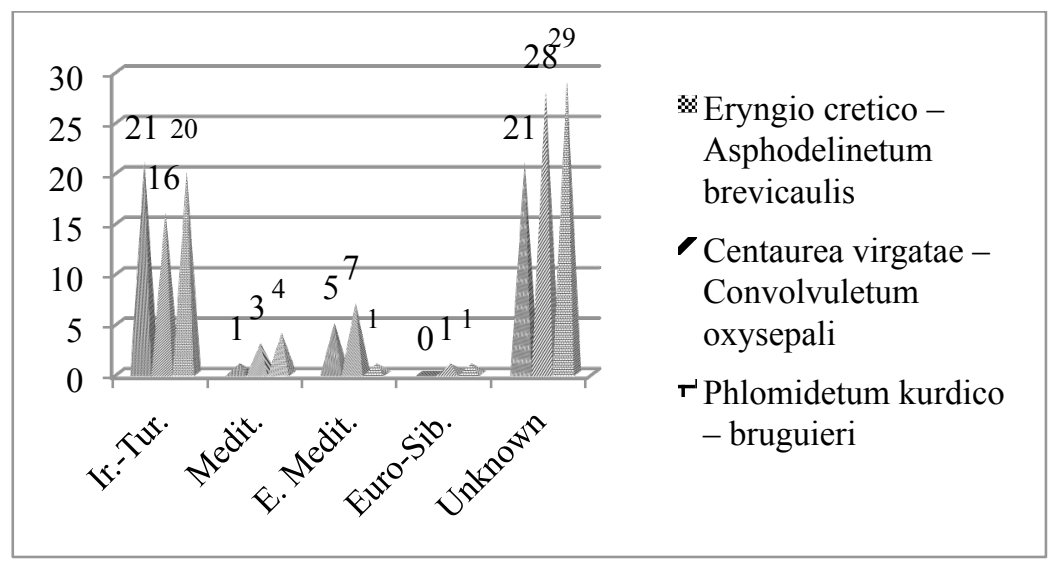

Figure 3. Chrology spectrum of associations.

When the life forms of identified taxa are analysed in accordance with Raunkiaer (1934) methods, the dominance of hemicryptophytes is understood. This result supports the opinion alleges that Irano - Turanian phytogeographic region is characterized by hemicryptophytes and chamaephytes (Zohary 1973) (Figure 4).

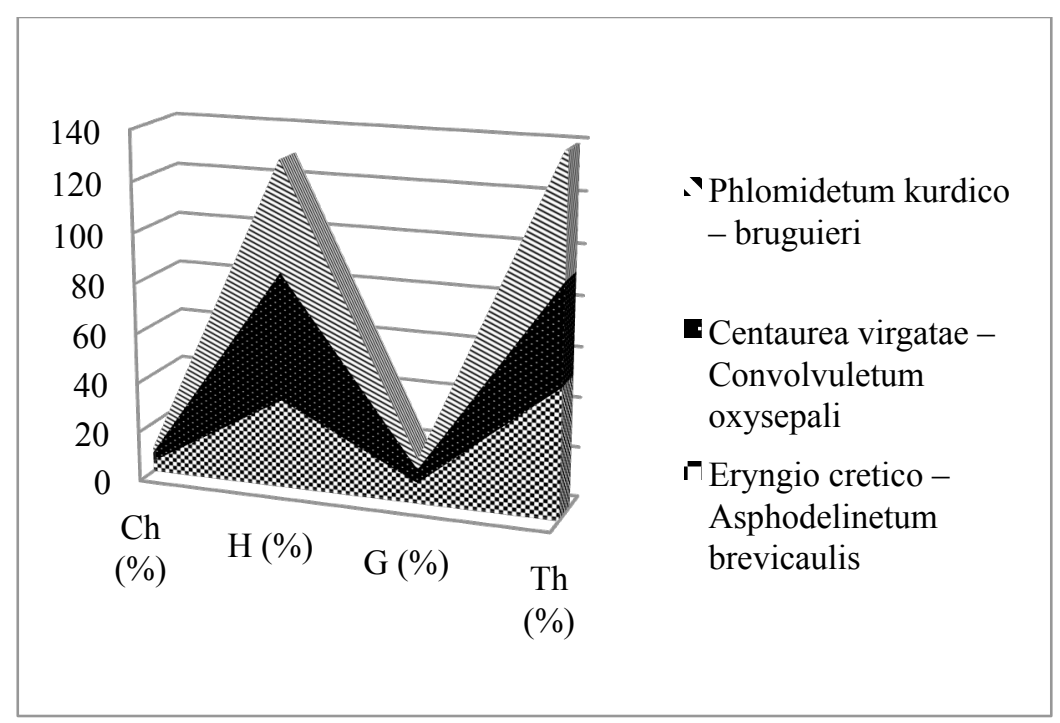

Figure 4. Life forms of associations (Ch: chamaephytes, H: hemicryptophytes, G: geophytes, Th: therophytes). 
As a conclusion of the phytosociological research conducted on the study area, the identified syntaxa of steppe vegetation, and their upper syntaxonomic units are shown below:

Class: Astragalo microcephali - Brometea tomentelli Quézel 1973

Order: Onobrychido armenae - Thymetalia leucostomi Akman, Ketenoğlu \& Quézel 1985

Alliance: Astragalo erythrotaeni-Gundelion armatae Kaya \& Ketenoğlu 2010

Association: Eryngio cretico - Asphodelinetum brevicaulis ass.nova

Association: Centaurea virgatae - Convolvuletum oxysepali ass.nova

Association: Phlomidetum kurdico - bruguieri Kaya 2011

\section{ACKNOWLEDGEMENTS}

The author thanks Prof. Dr. A. Selçuk Ertekin for help to identify the taxa.

\section{REFERENCES}

1. Akman Y., Ketenoğlu O. and Quézel P. 1985, A new syntaxon from central Anatolia. Ecologia Mediterranea, $11(2 / 3), 111-121$.

2. Anonymous, 1986, Büyük larousse (Güneydoğu Anadolu maddesi) Vol 8. Gelişim Yayınları, İstanbul, 4855-4856 s.

3. Anonymous, 2010, Şanlıurfa meteoroloji istasyonu rasat verileri. DMİ Genel Müdürlüğü Arşivi, Ankara.

4. Anonymous, 2011, Şanlıurfa il çevre durum raporu, Şanlıurfa Valiliği Çevre ve Şehircilik İl Müdürlügüü, Şanlıurfa, 206 s.

5. Braun-Blanquet J., 1965, Plant sociology (translated by Fuller G.D. \& Conard H.S.), McGraw-Hill, New York, $439 \mathrm{p}$.

6. Davis P.H. (ed.) 1965-1985, Flora of Turkey and the East Aegean Islands vol. 1-9, Edinburgh University Press, Edinburgh.

7. Davis P.H., Mill R.R. and Tan K. (eds.) 1988, Flora of Turkey and the East Aegean Islands (supplement) vol. 10, Edinburgh University Press, Edinburgh.

8. Eken G., Bozdoğan M., İsfendiyaroğlu S., Kılıç D.T. and Lise Y., 2006, Türkiye'nin önemli doğa alanları (Vol II), Doğa Derneği, $638 \mathrm{~s}$

9. Eyüpoğlu F., 1999, Türkiye topraklarının verimlilik durumlar1, Toprak ve Gübre Araştırma Enstitüsü Yayınları, Genel Yayın No: 220, Teknik Yayın No:T-67, Ankara.
10. Frey W. and Lösch R. 1998, Lehrbuch der geobotanik. Pflanze und Vegetation in Raum und Zeit. Gustav Fischer Verlag, Stuttgart, $436 \mathrm{p}$.

11. Gedikoğlu İ. 1990. Laboratuar analizlerinin gübre önerilerinde kullanılması ve halen kullanılan kriterler, Şanlıurfa Araştırma Enstitüsü Müdürlüğü Yayınları. Genel yayın No: 57, Teknik Yayın No:13, Şanlıurfa.

12. Güner A., Özhatay N., Ekim T. and Başer K.H.C., 2000, Flora of Turkey and the East Aegean Islands (supplement 2) vol. 11, Edinburgh University Press, Edinburgh.

13. Kaya, Ö.F. and Ertekin, A.S., 2009, Flora of the protected area at the Tek Tek Mountains (ŞanlıurfaTurkey), Ot Sistematik Botanik Dergisi, 16 (2), 79-96.

14. Kaya Ö.F., 2010a, Kaşmer dağı (Şanlıurfa)'nın step vejetasyonu üzerine sintaksonomik bir çalışma, Kastamonu Üniversitesi Orman Fakültesi Dergisi, 10 (1), 1-11.

15. Kaya Ö.F. and Ketenoğlu, O., 2010b, A syntaxonomical and synecological research on the steppe vegetation of the Karacadağ mountain (Şanlıurfa/Diyarbakır), Ecologia Mediterranean, 36 (1), 45-62.

16. Kaya, Ö.F., 2011a, New steppic syntaxa from southeastern Anatolia (Şanlıurfa, Turkey), Acta Botanica Gallica, 158 (2), 189-204.

17. Kaya, Ö.F., Ketenoğlu, O. and Yetim, S., 2011b, A synecological and syntaxonomical research of the secondary vegetation caused by overgrazing on Arat Mountain (Şanliurfa, Turkey), Acta Botanica Gallica, 158 (1), 13-25.

18. Quézel P., 1973, Contribution a l'etude phytocoenologique du masif du Taurus, Phytocoenologia, 1 (2), 131-222.

19. Raunkiaer C., 1934, The life forms of plants and statistical plant geography, Clarendon Press, Oxford, 632 p.

20. Sørensen T., 1948, A method of establishing groups of equal amplitude in plant sociology based on similarity of species content, Biol. Skr. K. Dan. Vidensk. Selsk., 5 (4), $1-34$.

21. Tüzüner A., 1990, Toprak ve su analiz laboratuarı el kitabı, Tarım Orman ve Köy İşleri Bakanlığı KHG Müdürlüğü Yayını, Ankara, 374 s.

22. Weber H.E., Moravec J. and Theurillat J.P., 2000, International code of phytosociological nomenclature $3^{\text {rd }}$ edition, Journal of Vegetation Science, 11, 739-768.

23. Yeniyurt C., Tatar B., Çetin T., Akarsu F., Altun C., Ataol M. and Körbalta H., 2009, Şanlıurfa Yaban Hayatı Geliştirme Sahası Gelişme ve Yönetim Planı 2010-2014, Doğa Derneği, Ankara, $55 \mathrm{~s}$. 